\title{
Improvement of recombination efficiency by mutation of Red proteins
}

\author{
Manabu Nakayama ${ }^{1,2}$ and Osamu Ohara ${ }^{1-3}$
}

BioTechniques 38:917-924 (June 2005)

\begin{abstract}
The Red recombination system of the bacteriophage $\lambda$ is a useful tool for engineering bacterial artificial chromosomes (BACs) because desired mutations can be made in any part of the DNA, even in large DNAs, independent of the presence of appropriate restriction enzyme sites. Many have tried changing the inducible promoter or decreasing the copy number of Red genes in Escherichia coli to improve the recombination efficiency. Here we describe a novel method for increasing the recombination efficiency of the Red system by mutating the Red proteins. Mutant Red $\alpha$ and Red $\beta$ independently increased recombination efficiency. We found that the 18-bp 5' untranslated region (UTR) upstream from the ATG initiation codon of Gam protein is important for recombination efficiency. Although low rates of recombination have been observed in systems using high copy number plasmids, when we performed Red recombination using a high copy number plasmid containing $5^{\prime}$-UTR, mutant Red $\alpha$, and mutant Red $\beta$, recombination increased approximately 145-fold. The novel possibility that mutant Red $\alpha$ and Red $\beta$ can increase recombination efficiency indicates a new direction for improving recombination efficiency of expression systems that use low or high copy number plasmids or a defective prophage integrated into the E. coli chromosome.
\end{abstract}

\section{INTRODUCTION}

Bacterial artificial chromosomes (BACs) can stably maintain large segments of foreign DNA (approximately $150 \mathrm{~kb}$ ) and are thus powerful tools for generating long-range physical maps and for sequencing whole genomes. At the beginning of the postgenome sequencing era, BAC systems have become an equally powerful tool for comprehensive functional genomics. For example, BACs have been used as expression systems that contain large introns and exons (1), and modified BAC clones have been used to introduce lengthy homologous DNA segments into mouse embryonic stem (ES) cells (2). For the BAC system to be used effectively in all aspects of functional genomics and not only for genome sequencing, it is important to be able to freely manipulate and modify BAC clones containing large DNA inserts. To date, traditional restriction enzyme-ligation methods have been used to prepare targeting vectors for generating knockout mice. It is very difficult, however, to insert suitable marker genes, such as neo (neomycin) and $t k$ (thymidine kinase), into large DNA segments contained within these clones using traditional methods because appropriate restriction enzyme site(s) are not always located in suitable places within the BAC clone. Thus, with traditional restriction enzyme methods, the location and size of the deletion within the targeted gene as well as the correct positioning of a translational frame fused to a reporter gene often depend on and are limited by the presence and position of restriction enzyme sites. Because constructing conditional knockout and knockin mice will increasingly necessitate the construction of more complicated targeting vectors (3), researchers will need a method that allows them to freely modify DNA segments within BAC clones. The Red recombination system promises to be a useful tool in this regard because specific sites within this system can be readily modified with ease and accuracy.

Only three genes are required to carry out recombination using the Red system (4). The exo gene encodes the
Red $\alpha$ protein, a $5^{\prime}$ to $3^{\prime}$ exonuclease that acts on the $5^{\prime}$ ends of a linear double-stranded DNA (dsDNA) fragment to produce $3^{\prime}$ single-stranded DNA (ssDNA) overhangs. The bet gene encodes a pairing protein $(\operatorname{Red} \beta)$ that binds to the $3^{\prime}$ ssDNA overhangs created by the Red $\alpha$ protein and promotes annealing. The recombination function of Red $\alpha$ and $\operatorname{Red} \beta$ proteins are further assisted by the $\lambda$-encoded Gam protein, which inhibits the RecBCD exonuclease activity of Escherichia coli, thus preventing the degradation of linear DNA fragments. Red-mediated homologous recombination occurs via a short, approximately 50-bp long homologous region shared by the two molecules that are to be recombined. Because the sequence of these homologous regions can be chosen freely, any part of the target molecule can be specifically altered.

Some have attempted to increase the rate of the Red recombination system using short (40-60 bp) homologous DNAs, but not long homologous arms (200-500 bp) (5). One group (6-8) changed the standard arabinose-

\footnotetext{
${ }^{1}$ Kazusa DNA Research Institute, Kisarazu, Chiba, ${ }^{2}$ Chiba University, Kisarazu, Chiba, and

${ }^{3}$ The Institute of Physical and Chemical Research (RIKEN), Yokohama, Kanagawa, Japan
} 
inducible promoter, which directs the expression of Red proteins, to a strong promoter that mediates continuous expression of bet and gam. They also employed an expression system based on low copy number plasmids depending on the oriR101. Another group $(9,10)$ integrated a defective $\lambda$ prophage into the $E$. coli chromosome. In the defective prophage system, the expression of exo, bet, and gam genes is directed by the strong $\lambda \mathrm{P}_{\mathrm{L}}$ promoter, which itself is under the control of the temperaturesensitive $\lambda$ cI 857 repressor. At $32^{\circ} \mathrm{C}$, the expression of exo, bet, and gam is undetectable. However, by shifting the culture temperature to $42^{\circ} \mathrm{C}$ for $15 \mathrm{~min}$, the expression of $\operatorname{Red} \alpha, \operatorname{Red} \beta$, and Gam is induced to very high levels.

When the Red system is used to modify BAC clones, it is convenient to use plasmids derived from pBR322 of ColE1 origin because they are compatible with BAC vectors of $F$ factor origin and because they can maintain high copy numbers and be handled easily and conveniently. When used in conjunction with high copy number plasmids, however, the recombination efficiency of the Red system with PCR-generated substrates is generally low (4). One possible reason is that the inhibition of RecBCD by Gam causes the formation of plasmid multimers, which decreases the frequency of recombination.

Although the Red recombination system is very useful for the flexible modification of BAC clones using only short 40-60 bp homologous fragments, one weakness of this system is that its recombination efficiency is still too low for routine use by nonspecialists. To further increase the efficiency of Red recombination, we propose a qualitatively different method from the previous approaches of changing promoters and copy number of expression units. To achieve our goal, it is necessary to examine in detail the factors that affect recombination efficiency. Thus, in the current study, we analyzed factors that influence the efficiency of Red recombination. We identified mutants of Red $\alpha$ and $\operatorname{Red} \beta$ that improved the efficiency of Red recombination. We also describe the importance of the $5^{\prime}$ untranslated region (UTR) adjacent to Gam for increasing homologous recombination.

\section{MATERIALS AND METHODS}

\section{Construction of Plasmids Supplying $\operatorname{Red} \alpha, \operatorname{Red} \beta$, and Gam Proteins}

We constructed plasmid pBADTc by replacing the ampicillin resistance gene of pBAD/HisA (Invitrogen, Carlsbad, CA, USA) with the tetracycline resistance gene of pTRG (Stratagene, La Jolla, CA, USA). Oligomers (5'-CCAAGCTTCGCGACTAGTTTAAACCTGCAGGCGGCCGCTCAGGTCGAGGTGGCCCGGCTCCAT-3' and 5'-GGAAGCTTCATGTTTGACAGCTTATCATCG-3') were used for the PCR amplification of the tetracycline resistance gene. The resulting PCR product was digested with HindIII and blunted. We ligated the DNA fragment onto the blunted $B s p \mathrm{HI}$ fragment of $\mathrm{pBAD} / \mathrm{His}$. For the construction of pBADTcRed4, we used upstream primer 3 (5'-CGCCATG GATAT TA ATAC T G A A ACTGAGATC-3') and downstream primer 4 (5'-CCAAGCTTCATCGCCATTGCTCCCCAAATAC-3') to PCR amplify the Gam-Red $\beta$-Red $\alpha$ operon from the $\lambda$ phage clind ts 857 Sam7 DNA (Invitrogen). The resulting PCR product was digested with $\mathrm{NcoI}$ and HindIII, and then ligated into the NcoI-HindIII site of pBADTc. For the construction of pBADTcRed19, we used another upstream primer, primer 5'-CGTCGACGCTTATAAAAAATGG-3', for PCR amplification. The resulting PCR product was inserted into the $\mathrm{pGEM}^{\circledR}$-T Easy Vector (Promega, Madison, WI, USA). The plasmid was digested with $N c o$ I and SacI, and the NcoI-SacI DNA fragment was ligated into the NcoI-SacI site of pBADTc.

\section{Preparation of the neo/km Cassette}

Plasmid pMC1neoPolyA (Stratagene), which is often used for homologous recombination in mouse ES cells, contains the eukaryotic tk promoter and the neomycin resistance gene. The E. coli consensus promoter [-35 (TTGACA) and -10 (TATAAT)] was added upstream to the tk promoter of pMC1neoPolyA. The ShineDalgarno (SD)-kozak sequence $5^{\prime}$ AGGAGGCCACC-3' was positioned in front of the ATG initiation codon. The modified neo gene works well in both E. coli and mouse ES cells. We positioned two 52-bp sequences homologous to the gene of interest, site A (5'-AGAAAGGCCAACGCTGGGGGTCTCTGGAGCGCCGTGGGATGCAAGCTATGGA-3') and site B (5'-GGAGGATGAGCGGCTGGCCAGTGCCCAGCAAGCAGAAGTATTCACCAAGCAG-3'), at each end of the neo gene. Two BseRI sites were placed at each end of the neo $/ \mathrm{km}$ cassette to facilitate the removal of the cassette from the plasmid. The neo $/ \mathrm{km}$ cassette containing sites A and B was removed by digesting the plasmid with restriction endonuclease $B s e$ RI. Because the recognition site of $B s e$ RI is GAGGAG(10/8), a nonpalindromic sequence, and because $B s e$ RI cleaves at a distance from its recognition site, the resulting neo/ $\mathrm{km}$ cassette had no nucleotide overhangs that might inhibit Red recombination. After digestion with $B s e$ RI, we recovered the neo $/ \mathrm{km}$ cassette following agarose gel electrophoresis and purified it using the Wizard ${ }^{\circledR}$ SV Gel and PCR Clean-Up System (Promega). The neo/km cassette was extracted with phenol/chloroform, precipitated with ethanol, and dissolved in TE (Tris-EDTA) buffer.

\section{SDS-PAGE Analysis of Red Proteins}

E. coli DH10B strain (Invitrogen) was transformed with either pBADTcRed4 or pBADTcRed4 derivatives. Transformants were grown on Luria-Bertani (LB) agar plates containing tetracycline $(12.5 \mu \mathrm{g} / \mathrm{mL})$. The cultures were grown overnight from a single colony, diluted 100 -fold in $2 \mathrm{~mL} \mathrm{LB}$ medium containing tetracycline $(12.5 \mu \mathrm{g} / \mathrm{mL})$, and then grown for $1.5 \mathrm{~h}$ to an $\mathrm{A}_{600}$ of 0.1 . Twenty microliters of $10 \%$ L-arabinose (final concentration of $0.1 \%$ ) were added to $2 \mathrm{~mL}$ culture to induce $\operatorname{Red} \alpha, \operatorname{Red} \beta$, and Gam expression. The culture was incubated at $37^{\circ} \mathrm{C}$ for $2 \mathrm{~h}$ under gentle agitation. $E$. coli cells were centrifuged for $30 \mathrm{~s}$ at $14,000 \times g$ at $4^{\circ} \mathrm{C}$, washed with $1 \mathrm{~mL}$ ice-cold phosphate-buffered saline (PBS), and resuspended in 300 $\mu \mathrm{L}$ PBS. These cells were subsequently lysed with a sonicator and centrifuged, and aliquots of soluble fractions were 
loaded onto $12.5 \%$ polyacrylamide gels and subjected to sodium dodecyl sulfate polyacrylamide gel electrophoresis (SDS-PAGE). The gels were stained with Coomassie ${ }^{\circledR}$ brilliant blue (Wako Pure Chemical Industries, Ltd., Osaka, Japan).

\section{Red Recombination}

E. coli $\mathrm{DH} 10 \mathrm{~B}$ strain (Invitrogen) containing a BAC of interest was transformed with either pBADTcRed4 or pBADTcRed4 derivatives by electroporation, according to a standard procedure (11). Transformants were grown on LB agar plates containing tetracycline and chloramphenicol (12.5 $\mu \mathrm{g} / \mathrm{mL}$ each). For BAC modification, cultures containing BACs were grown overnight from a single colony, diluted 100-fold in $2 \mathrm{~mL} \mathrm{LB}$ medium containing tetracycline and chloramphenicol (12.5 $\mu \mathrm{g} / \mathrm{mL}$ each), and then grown for $1.5 \mathrm{~h}$ to an $\mathrm{A}_{600}$ of 0.1 . We added $2 \mathrm{~mL}$ culture to $20 \mu \mathrm{L}$ of $10 \%$ L-arabinose (final concentration of $0.1 \%$ ) to induce $\operatorname{Red} \alpha, \operatorname{Red} \beta$, and Gam expression. The culture was incubated at $37^{\circ} \mathrm{C}$ for $1 \mathrm{~h}$ under gentle agitation. When an $\mathrm{A}_{600} 0.4$ confluency of $E$. coli cells was achieved, they were centrifuged for $30 \mathrm{~s}$ at $8000 \times g$ at $2^{\circ} \mathrm{C}$, washed gently with $1 \mathrm{~mL}$ ice-cold sterile water and $1 \mathrm{~mL}$ ice-cold sterile $10 \%$ glycerol twice, and resuspended in $25 \mu \mathrm{L}$ ice-cold sterile $10 \%$ glycerol. Ice-cold competent cells $(25 \mu \mathrm{L})$ and linear neo/km DNA cassettes (250 ng) were combined into $0.1 \mathrm{~cm}$ cuvettes, and cell transformation was performed by electroporation using a Gene Pulser ${ }^{\circledR}$ II (Bio-Rad Laboratories, Hercules, CA, USA) set at $1.8 \mathrm{kV}$ and at $25 \mu \mathrm{F}$ with a pulse controller set to $200 \mathrm{ohms}$. One milliliter of SOC medium (Invitrogen) was added to the culture after electroporation. Following incubation at $37^{\circ} \mathrm{C}$ for $80 \mathrm{~min}$ under gentle agitation, the cells were spread onto LB agar plates containing kanamycin $(25 \mu \mathrm{g} / \mathrm{mL})$ and chloramphenicol (12.5 $\mu \mathrm{g} / \mathrm{mL})$, incubated for an additional $16 \mathrm{~h}$, and drug-resistant colonies were counted. The recombination efficiency of the Red system was calculated by averaging the total number of surviving colonies in three independent experiments. Recombination was confirmed in 10-20 clones by using PCR with primers of BAC clone sequences located outside the identical $\mathrm{A}$ and $\mathrm{B}$ regions (Figure 1). These analyses revealed that over $95 \%$ of the clones correctly recombined.

\section{Random PCR Mutagenesis and Screening of Clones Displaying a High Rate of Red Recombination Activity}

We performed PCR-based random mutagenesis using primers 3 and 4 according to a method previously described (12). For screening mutant Red $\alpha$, the mutagenized PCR product was digested with EcoRI and HindIII and ligated into the EcoRI-HindIII site of pBADTcRed4. The ligated sample was transformed into E. coli DH10B, and then grown on LB plates containing $12.5 \mu \mathrm{g} / \mathrm{mL}$ tetracycline. Tetracycline-resistant colonies were pooled, and the plasmids were purified with a High-Purity Plasmid Midiprep System (Marlingen Bioscience, Ijamsville, MD, USA). The purified plasmid library

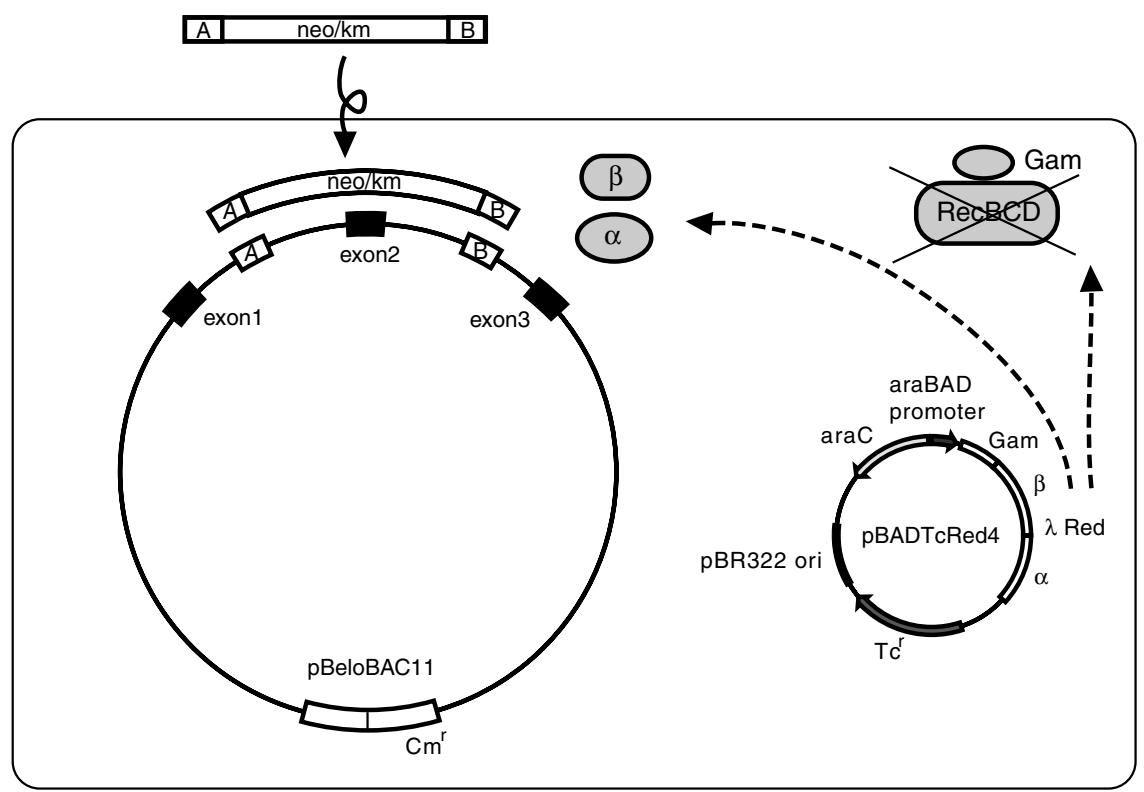

Figure 1. Map of plasmid used for Red recombination and diagram illustrating the principle for modifying BACs. An operon containing the $\lambda$ phage gam, bet, and exo genes was positioned behind the $\mathrm{L}$-arabinose-inducible $\mathrm{BAD}$ promoter. The ampicillin resistance gene was replaced with the tetracycline resistance gene. Panels A and B represent the 52-bp sequences of the neo $/ \mathrm{km}$ cassette that are identical to targeted sequences contained within the BAC clone. The neo/km cassette works well for selection in both Escherichia coli and mouse ES cells. Electroporation was used to introduce the neo/ $/ \mathrm{km}$ cassette into $E$. coli $\mathrm{DH} 10 \mathrm{~B}$ containing the BAC clone and a high copy number plasmid supplying $\operatorname{Red} \alpha, \operatorname{Red} \beta$, and Gam proteins. BAC, bacterial artificial chromosome; ES, embryonic stem. 
bination was performed. We selected kanamycin- and chloramphenicolresistant colonies and identified the DNA sequence of mutated regions. For screening mutant $\operatorname{Red} \beta$, the mutagenized PCR product was digested with SalI and EcoRI and ligated into the SalI-EcoRI site of pBADTcRed4. The same steps described above were repeated.

\section{RESULTS}

Because our BAC clone had an $\mathrm{F}$ factor origin, we chose a compatible, high copy number plasmid system, $\mathrm{pBAD} / \mathrm{HisA}$, as the backbone to supply the Red and Gam proteins required for homologous recombination. We did not choose the pSC101 plasmid because it is incompatible with a BAC clone and is a low copy number plasmid. Furthermore, we preferred the Red recombination system in a high copy number plasmid to that of an integrated form in the E. coli chromosome because it is more convenient and simpler to manipulate each component of the Red recombination system using a high copy number plasmid system.

To construct our gene targeting vector using this Red recombination

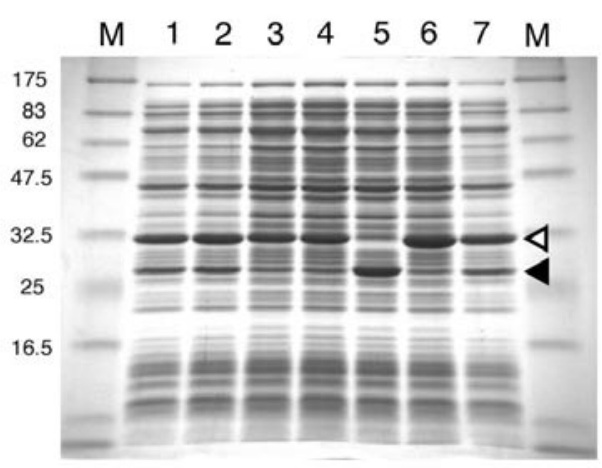

Figure 2. Expression of Red proteins after arabinose induction. Lanes 1 and 2: soluble fraction of Escherichia coli containing pBADTcRed 4 two hours after arabinose induction (two independent colonies). Lanes 3 and 4: soluble fraction of $E$. coli containing pBADTcRed19 two hours after arabinose induction (two independent colonies). Lane 5: soluble fraction of E. coli producing only Red $\alpha$. Lane 6: soluble fraction of E. coli producing only Red $\beta$. Lane 7: soluble fraction of $E$. coli containing pBADTcTypeF two hours after arabinose induction. Lane M, molecular weight size marker (Prestained Protein Marker; New England Biolabs, Beverly, MA, USA). Shaded and unshaded triangles point to $\operatorname{Red} \alpha$ and $\operatorname{Red} \beta$, respectively. system, we planned to introduce two cassettes, one containing the neo gene (for neomycin selection) and one containing the $t k$ gene (for negative selection), into an intact BAC clone. This requires the use of markers. Initially, when Red recombination was performed using the tetracycline resistance gene as a selection marker, we encountered a significantly lower number of surviving colonies after recombination than when using kanamycin and ampicillin resistance genes. To increase the total number of surviving colonies after recombination, we constructed a new plasmid replacing the ampicillin resistance gene with the tetracycline resistance gene. Steele et al. (13) observed an approxate 100 -fold reduction in the transpBR322 when induced by electroporation but not by $\mathrm{CaCl}_{2}$, following selection with tetracycline rather than picillin. We concluded the same the low number of surviving colonies after recombination that we initially bserved may have occurred because of the low electroporation rates that occur with substrates containing the tetracycline resistance gene as the selectable marker.

Using a similar approach to that used by Muyrers et al. (6), we constructed a vector capable of expressing the two Red gene products and the Gam gene product of $\lambda$ phage. We inserted the gam, bet, and exo genes, in this order, into pBADTc in the form of an operon located between the ATG initiation codon and the transcriptional terminator of $\mathrm{rrnB}$ (Figure 1). As with pBAD/HisA, pBADTc can be induced to express foreign gene products by treating cells with arabinose. A neo $/ \mathrm{km}$ cassette contains neomycin and kanamycin resistance genes directed by both prokaryotic and eukaryotic promoters. This cassette also contains 52 bp DNA sequences (sites A and B) that are identical to the sequences of targeted sites of a gene in the BAC clone (Figure 1). After inducing expression of the Red gene products with arabinose, we performed Red recombination by introducing the neo $/ \mathrm{km}$ cassette into E. coli containing the BAC clone. We recovered approximately 20 colonies using both kanamycin and chloramphenicol selection plates (the BAC vector confers chloramphenicol resistance; the recombined BAC confers both kanamycin and chloramphenicol resistance). Detailed analysis revealed that these colonies recombined exactly at the expected target site. However, the rate of recombination was too low to warrant the use of this technique for routine modification of BAC clones. DNA sequencing of pBADTcRed4 confirmed that the gam, bet, and exo genes of $\lambda$ phage inserted correctly. Moreover, no mutations were detected in pBADTcRed4. SDS-PAGE assessment for Red gene expression detected large amounts of Red $\alpha$ and $\operatorname{Red} \beta$ proteins but no Gam protein after induction with arabinose (Figure 2 ); these proteins were not observed before induction (data not shown), indicating that either Gam is a small protein and/or a small quantity of Gam was expressed (Figure 2). Nonetheless, this finding indicated that there was a possible problem in the expression of Gam protein by pBADTcRed4.

Generally, when constructing expression vectors for efficient translation, a functional ribosome binding site containing an SD sequence is positioned $5^{\prime}$ to the ATG initiation codon (14). The typical SD sequence is AGGAGG. Placing the SD and ATG initiation codon $9 \pm 3$ nucleotides apart increases translation. In pBADTcRed4, the SD sequence of Gam protein, AGGAGG, is located 8 bp upstream of the ATG initiation codon. Although we initially expected the SD translational signal to produce sufficiently high levels of Red gene products in E. coli, the lack of Gam protein production from pBADTcRed4 led us to conclude that some innate translational signal in the $5^{\prime}$ untranslated sequence used by 
wild-type $\lambda$ phage may be necessary for Gam protein production. This prompted us to add an 18-bp 5'-UTR fragment to pBADTc. The 18-bp 5'UTR plus Gam, Red $\beta$, and Red $\alpha$ were derived from PCR reamplification using $\lambda$ phage DNA as a template. Because PCR amplification may have induced mutations in the $\lambda$ phage DNA that could affect recombination, we also assessed the recombination efficiency of six additional clones using the same neo/km cassette and E. coli containing the BAC clone. The pBADTcRed19 clone showed the highest rate of recombination of the Red system, recombining at a rate approximately 100 times higher than that of pBADTcRed4 (Figure 3C). However, the recombination efficiency of the five other clones was lower, which suggests that other factor(s) influenced the rate of recombination in pBADTcRed19. DNA sequencing revealed that, unlike pBADTcRed4, pBADTcRed19 contained two mutations in addition to the presence of the 18-bp 5'-UTR. One mutation occurred in the Red $\alpha$ stop codon, causing an insertion of an additional 48 amino acids (RRILITSEFA AACRSTIWES SRSAAGTIWE FEAWLFWRMR EDFQPDTD) at the carboxyl terminus of the Red $\alpha$ protein. The other mutation occurred in $\operatorname{Red} \beta$, resulting in a nonsynonymous amino acid substitution (Cys-to-Arg at position $118, \mathrm{C} 118 \mathrm{R})$ in the $\operatorname{Red} \beta$ protein (Figure 3 ). These data provide the first evidence that mutations in Red $\alpha$ and $\operatorname{Red} \beta$ proteins can increase the recombination efficiency of Red recombination. Thus, to determine which of the two mutations and/or $18 \mathrm{bp}$ of $5^{\prime}$-UTR contributes most to increasing recombination efficiency, we constructed chimeric clones containing various combinations of these mutations and 18 bp $5^{\prime}$-UTR (Figure 3B).

For TypeA and TypeB clones, which contain either mutant Red $\alpha$ protein or mutant $\operatorname{Red} \beta$, respectively, recombination efficiency increased approximately 2 -fold. For the TypeC clone, which contains both mutant Red $\alpha$ and $\operatorname{Red} \beta$ proteins, efficiency increased approximately 4-fold, indicating that, because of this additive effect, mutant
A

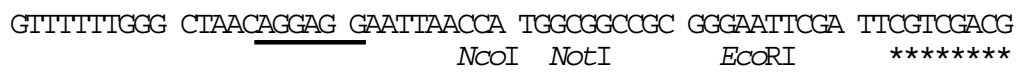

CITATAAAAA ATGGATATTA ATACTGAAAC TGAGATCAAG CAAAAGCATT CACTAACCCC $\begin{array}{llllllllllllllllll}\star \star \star * * * * * * * & M & D & I & N & T & E & T & E & I & K & Q & K & H & S & L & T & P\end{array}$

B

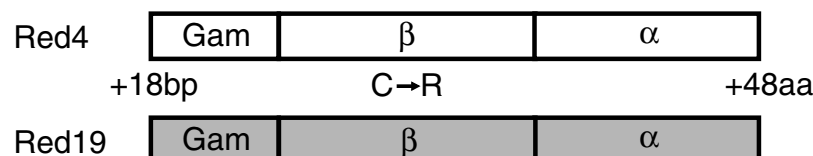

\begin{tabular}{l|l|l|}
\hline & & \\
\hline
\end{tabular}

\begin{tabular}{|c|c|c|c|}
\hline TypeB & Gam & $\beta$ & $\alpha$ \\
\hline
\end{tabular}

\begin{tabular}{|c|c|c|c|}
\hline TypeC & Gam & $\beta$ & $\alpha$ \\
\hline TypeD & Gam & $\beta$ & $\alpha$ \\
\hline
\end{tabular}

\begin{tabular}{c|c|c|}
\hline TypeE & $\beta$ & $\alpha$ \\
\hline \multicolumn{2}{|c|}{ Gam } & \multicolumn{1}{c|}{$\alpha$} \\
\hline
\end{tabular}

\begin{tabular}{|c|c|c|c|}
\hline TypeF & Gam & $\beta$ & 0. \\
\hline \multicolumn{2}{|c|}{$+18 b p$} & $C \rightarrow R$ & $R \rightarrow Q$ \\
\hline 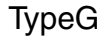 & Gam & $\beta$ & ar \\
\hline
\end{tabular}

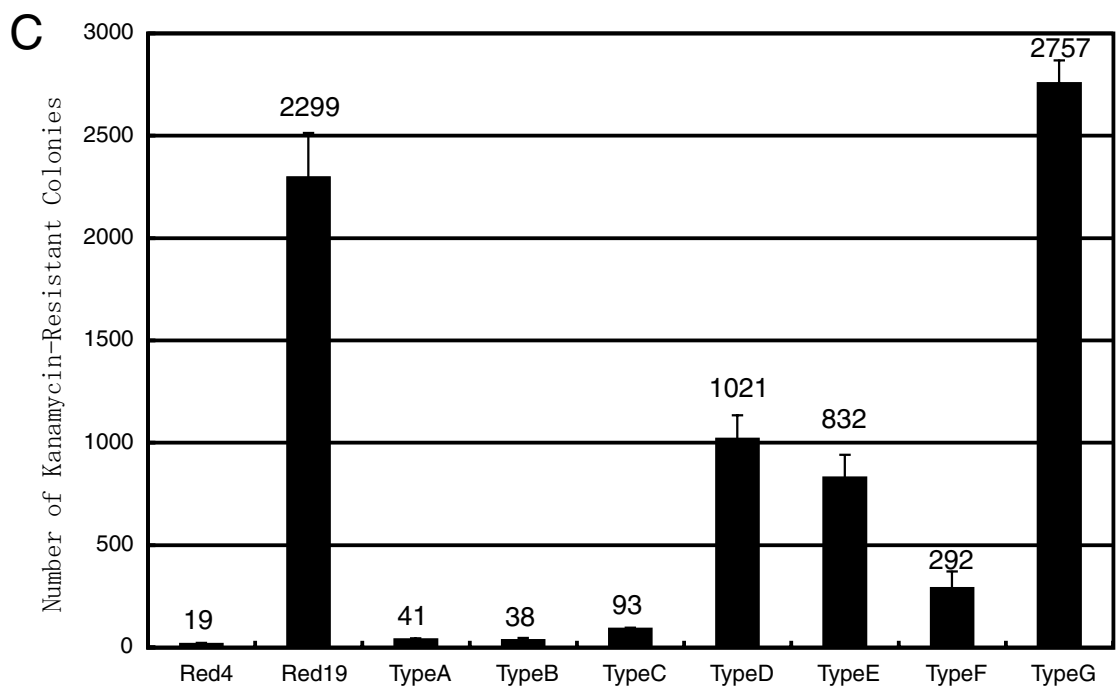

Figure 3. Addition of 18 bp 5'-UTR and mutant protein increases the frequency of Red recombination. (A) Sequence of translational regulation region and initiation codon of Gam protein. Underline indicates typical SD sequence. Asterisks show 18 bp 5'-UTR of gam gene derived from wild-type $\lambda$ phage. The bolded sequences represent the start codon. In pBADTcRed4, the ATG initiation codon was ligated into the $\mathrm{NcoI}$ site. (B) Illustrations of various chimeric constructs of Red recombination components. The +18 bp indicates that the 18 bp 5'-UTR of gam gene was added. Mutant Red $\beta$ contains a C118R substitution. The "+48aa" indicates that the stop codon of Red $\alpha$ was converted and an additional 48 amino acids was inserted to the carboxyl terminus of Red $\alpha$ protein. Constructs TypeF and TypeG contain mutant Red $\alpha$ with a R137Q substitution. Unshaded, shaded, and striped rectangles represent Red recombination components derived from pBADTcRed4, pBADTcRed19, and pBADTcTypeF, respectively. (C) Efficiency of Red recombination of various constructs. The vertical axis represents the number of kanamycin-resistant colonies following Red recombination (see Materials and Methods). Using PCR with primers of BAC clone sequences located outside the identical regions A and B (compare with Figure 1), we assessed 10-20 clones and confirmed that over $95 \%$ of these clones correctly recombined. UTR, untranslated region; SD, Shine-Dalgarno; BAC, bacterial artificial chromosome. 
Red $\alpha$ and $\operatorname{Red} \beta$ proteins act independently. For TypeC, TypeD, and TypeE clones, recombination efficiency decreased by approximately 25 -fold, 2.3-fold, and 2.8-fold, respectively, compared to that of pBADTcRed 19 . TypeC contained both mutant Red proteins but no $5^{\prime}$-UTR, TypeD contained mutant $\operatorname{Red} \beta$ and $5^{\prime}$-UTR, and TypeE contained mutant Red $\alpha$ and $5^{\prime}$-UTR. From these results, it is clear that the 5'-UTR of Gam increases recombination efficiency the most at 25-fold, whereas mutant Red $\alpha$ and $\operatorname{Red} \beta$ increase recombination efficiency approximately 2 -fold each.

To determine how mutations in Red $\alpha$ and $\operatorname{Red} \beta$ proteins affect the amounts of Red protein produced by $E$. coli after arabinose induction, we performed SDS-PAGE analysis of the Red protein produced from bacteria transformed with pBADTcRed19. Because Red $\alpha$ protein from pBADTcRed19 had an extra 48 amino acids, its size is expected to be $5.7 \mathrm{kDa}$ larger than that of the intact Red $\alpha$ protein. Its expression levels, however, were undetectable for unknown reasons, perhaps because of instability of the Red $\alpha$ protein containing the extra 48 amino acids. The amount of Red $\alpha$ protein from pBADTcRed 19 significantly decreased compared to the amount produced from pBADTcRed4. The amount of $\operatorname{Red} \beta$ protein from pBADTcRed19, on the other hand, was almost that same as that from pBADTcRed4 (Figure 2).

These results suggest that other types of mutant $\operatorname{Red} \alpha$ and $\operatorname{Red} \beta$ proteins may also increase recombination efficiency significantly. To test whether this is indeed the case, we first constructed a clone library of mutant $\operatorname{Red} \alpha$ and $\operatorname{Red} \beta$ proteins using pBADTcRed4 and random PCR mutagenesis with dITP. We then screened for clones displaying high rates of recombination by transforming these mutant Red protein clones into E. coli harboring the $\mathrm{BAC}$ clone and performing the Red recombination reaction with the neo $/ \mathrm{km}$ cassette. Next, we selected kanamycin-resistant colonies and concentrated mutant Red $\alpha$ and $\operatorname{Red} \beta$ protein clones displaying efficient Red recombination by purification. Plasmids supplying the mutant Red proteins were reintroduced into
E. coli containing the BAC clone, and we screened for clones displaying high rates of recombination by performing the Red recombination reaction again. Using this method of kanamycin resistance selection and two cycles of Red recombination, we were able to obtain an increasing number of clones that consistently displayed high rates of recombination. To assess the role of mutant Red $\alpha$ protein in promoting recombination, we sequenced these plasmids and identified three clones from the 10 colonies we tested that possessed a mutation in Red $\alpha$ resulting from a nonsynonymous amino acid substitution (R137Q; Figure 3C, TypeF). When Red recombination was performed using the TypeF plasmid, recombination efficiency increased 15fold compared to that of pBADTcRed4 (Figure 3C). However, the recombination efficiency of the other clones was almost the same as that of pBADTcRed4. The recombination efficiency of clones possessing mutant $\operatorname{Red} \beta$ protein remained unchanged, even when two cycles of Red recombination were performed.

SDS-PAGE revealed that TypeF produced nearly the same amount of mutant Red $\alpha$ protein (data not shown) as did pBADTcRed4 (Figure 2). This is in stark contrast to pBADTcRed19, which produced less mutant Red $\alpha$ protein than did pBADTcRed4. These findings suggest that the mechanism by which recombination increases is different in cells transformed with TypeF compared to those transformed with pBADTcRed19. Thus, because the Red $\alpha$ protein of pBADTcRed19 increased recombination 2-fold, whereas the Red $\alpha$ protein of TypeF increased recombination 15 -fold, we expected a higher rate of Red recombination in systems using mutant Red $\alpha$ protein from TypeF than in systems using Red $\alpha$ protein from pBADTcRed19. As shown in Figure 3C, TypeG, which contains both mutant $\operatorname{Red} \beta$ and $\operatorname{Red} \alpha$ as well as $5^{\prime}$ UTR, increased recombination 1.2-fold compared to pBADTcRed19. Thus, the TypeG plasmid contains the combination of genes that promotes the most efficient Red recombination based on a high copy number plasmid.

\section{DISCUSSION}

Previous attempts to increase the efficiency of Red recombination mainly focused on changing the transcriptional promoter and copy number of expression units supplying Red proteins. This study is the first to report that mutant $\operatorname{Red} \alpha$ and $\operatorname{Red} \beta$ proteins increase the recombination efficiency of the Red recombination system. Mutant Red $\alpha$ (R137Q) and mutant $\operatorname{Red} \beta(\mathrm{C} 118 \mathrm{R})$ proteins increased the rate of recombination 2.5 -fold (compare TypeD and TypeG plasmids) and 2-fold, respectively. In addition, we found that the 18-bp 5'-UTR of $\lambda$ Gam also increases the rate of Red recombination. Modifying the translational control region of Gam by placing the 18 -bp $5^{\prime}$-UTR from wild-type $\lambda$ phage in front of the initiation ATG codon and the SD sequence 45 bp upstream of ATG (Figure 3A), we increased the efficiency of Red recombination in our high copy number plasmid system by 25 -fold. The importance of the $5^{\prime}$ UTR of Gam protein in promoting Red recombination has not been previously reported.

At the present time, we do not know exactly how the 18 -bp $5^{\prime}$-UTR from $\lambda$ phage affects recombination efficiency. One possibility is that the $\lambda 5^{\prime}$-UTR may increase recombination by decreasing Gam production. In pBADTcRed4, the typical SD sequence is optimally positioned upstream of ATG, thus ensuring the expression of sufficiently large amounts of Gam protein in $E$. coli harboring pBADTcRed4. We found recombination efficiency, however, to be greatly influenced by the viability of transformed $E$. coli, which in turn was influenced by Gam protein, which, in large amounts, is toxic to E. coli (15). Indeed, E. coli harboring pBADTcRed4 displayed slow growth rates (data not shown) and decreased recombination rates, which indicates that enough Gam was translated from pBADTcRed4 to affect, to some degree, E. coli viability and to decrease recombination. In contrast, E. coli harboring pBADTcRed19, a clone containing $\lambda 5^{\prime}$-UTR, displayed normal growth rates (data not shown) and increased recombination rates. We believe that the 18-bp 5'-UTR decreases 
Gam protein translation in E. coli by means of a $\lambda$ phage SD sequence, thus maintaining Gam production at sufficiently low levels, while preserving host viability and increasing recombination efficiency. The 18-bp 5'UTR sequence from $\lambda$ phage did not contain the typical SD sequence. This would explain why the recombination efficiency of pBADTcRed19 was high and that of pBADTcRed4 was low. At present, we have little information about the mechanism underlying the increased recombination efficiency caused by mutant $\operatorname{Red} \alpha$ and $\operatorname{Red} \beta$ proteins because substitutions identified in this study are not located in any previously characterized functional domains of $\operatorname{Red} \alpha$ and $\operatorname{Red} \beta$ proteins (16). An alternative interpretation of our findings is that both Red $\alpha$ mutations lower Red $\alpha$ activity relative to $\operatorname{Red} \beta$ activity. Indeed, when two Red proteins are expressed separately under the direction of promoters with different strengths, Muyrers and coworkers (7) observed that increasing the $\operatorname{Red} \beta /$ RecT-to-Red $\alpha / \operatorname{RecE}$ ratio increased the efficiency of Red/ET (RecE/RecT) recombination (7).

For the present study, we used a high copy number plasmid system because this system offers some advantages. When screening for mutants, as described here in this report, clone manipulation and identification by DNA sequencing become easier and more convenient when using high copy number plasmids because these plasmids result in higher yields. Another positive aspect of using high copy number plasmids is that many scientists, not just specialists accustomed to using plasmid systems, can easily adopt this method with a minimum of technical pitfalls compared to other methods that use unusual expression systems. During the preparation of our manuscript, pSC101-BAD-gbaA ${ }^{\text {tetra }}$, a plasmid developed previously for BAC modification, became commercially available throughout the world. This system uses pSC101, a low copy number plasmid, for supplying the two Red proteins and Gam protein for recombination (8). One potential problem with using pSC101BAD-gbaA ${ }^{\text {tetra }}$ to modify BACs is maintaining plasmid stability because both pSC101 and BACs (F factor origin) belong to the same incompatibility group (11). Using the pSC101BAD-gbaA ${ }^{\text {tetra }}$ system with our BAC clone and neo/km cassette according to the manufacturer's procedure, we were able to recover approximately 250 competent colonies (data not shown). Although the recombination efficiency of the pSC101-BAD-gbaA ${ }^{\text {tetra }}$ system does not preclude practical modification of BAC clones, its efficiency is lower than that of our system. In addition, the long-term effects of using pSC101 and BACs belonging to the same incompatibility group remain unknown.

In conclusion, we were able to make BAC modifications more easily with our high copy number plasmid system than with standard techniques. By using compatible BAC clones and pBR322-derived plasmids for introducing Red proteins into E. coli, we can simultaneously screen BACs of interest from a BAC library and modify these BACs for the construction of targeting vectors needed to prepare gene-targeted mice. At present, these two steps, screening BACs of mouse genomic DNA containing a gene of interest and constructing targeting vectors, are routinely performed separately. Because mouse genomic sequences available at public databases are now very accurate, we can now design two 50-60 base segments directly from these databases for the Red recombination system. In a single Red recombination reaction, one can simultaneously screen the BAC library and modify the BAC clone by introducing a neo $/ \mathrm{km}$ cassette into a targeted region of the gene of interest. Currently, many groups, including our own, screen BAC clones using 96- or 384-well plates and PCR amplification, which is a tedious, multistep task. It is especially very difficult to obtain a sufficient number of plates (with BAC clones contained within each of the 384 wells) for the initial step of this procedure. On the other hand, it is very easy to obtain a sufficient number of pooled samples from agar plates where thousands of BAC colonies can be grown. Using PCR, we can identify which pool contains the BAC clone of interest, and with one Red recombination reaction, we can simultaneously screen the BAC and modify it. The success of our preliminary experiments demonstrates that this strategy promises to be a more high-throughput method compared to previous methods. Finally, our novel finding that mutant Red proteins increase recombination efficiency can serve as the impetus for the systematic and large-scale screening of other potential mutants that can promote efficient recombination.

\section{ACKNOWLEDGMENTS}

We are grateful to E. Suzuki and S. Minorikawa for their excellent technical assistance. We also thank all our colleagues from the Kazusa cDNA Project Team for their cooperation. This study was supported by grants from the Kazusa DNA Research Institute and in part by Grants-in-Aid from the Ministry of Education, Culture, Sports, Science and Technology from the Japanese Government (to M.N.).

\section{COMPETING INTERESTS STATEMENT}

The authors declare no competing interests.

\section{REFERENCES}

1.Wade-Martins, R., E.R. Smith, E. Tyminski, E.A. Chiocca, and Y. Saeki. 2001. An infectious transfer and expression system for genomic DNA loci in human and mouse cells. Nat. Biotechnol. 19:1067-1070.

2.Yang, Y. and B. Seed. 2003. Site-specific gene targeting in mouse embryonic stem cells with intact bacterial artificial chromosomes. Nat. Biotechnol. 21:447-451.

3.Joyner, A.L. 2000. Gene Targeting: A Practical Approach. Oxford University Press, New York.

4.Murphy, K.C. 1998. Use of bacteriophage lambda recombination functions to promote gene replacement in Escherichia coli. J. Bacteriol. 180:2063-2071.

5.Liu, P., N.A. Jenkins, and N.G. Copeland. 2003. A highly efficient recombineering-based method for generating conditional knockout mutations. Genome Res. 13:476-484.

6.Muyrers, J.P., Y. Zhang, G. Testa, and A.F. Stewart. 1999. Rapid modification of bacterial artificial chromosomes by ET-recombination. Nucleic Acids Res. 27:1555-1557.

7.Muyrers, J.P., Y. Zhang, F. Buchholz, and A.F. Stewart. 2000. RecE/RecT and Redalpha/Redbeta initiate double-stranded break repair by specifically interacting with their respective partners. Genes Dev. 14:1971-1982. 
8.Zhang, Y., J.P. Muyrers, J. Rientjes, and A.F. Stewart. 2003. Phage annealing proteins promote oligonucleotide-directed mutagenesis in Escherichia coli and mouse ES cells. BMC Mol. Biol. 4:1.

9.Yu, D., H.M. Ellis, E.C. Lee, N.A. Jenkins, N.G. Copeland, and D.L. Court. 2000. An efficient recombination system for chromosome engineering in Escherichia coli. Proc. Natl. Acad. Sci. USA 97:5978-5983.

10.Lee, E.C., D. Yu, J. Martinez de Velasco, L. Tessarollo, D.A. Swing, D.L. Court, N.A. Jenkins, and N.G. Copeland. 2001. A highly efficient Escherichia coli-based chromosome engineering system adapted for recombinogenic targeting and subcloning of BAC DNA. Genomics 73:56-65.

11.Sambrook, J. and D.W. Russell. 2001. Molecular Cloning: A Laboratory Manual. CSH Laboratory Press, Cold Spring Harbor, NY.

12.Spee, J.H., W.M. de Vos, and O.P. Kuipers. 1993. Efficient random mutagenesis method with adjustable mutation frequency by use of PCR and dITP. Nucleic Acids Res. 21:777778.

13.Steele, C., S. Zhang, and E.J. Shillitoe. 1994. Effect of different antibiotics on efficiency of transformation of bacteria by electroporation. BioTechniques 17:360-365.

14.Balbas, P. and F. Bolivar. 1990. Design and construction of expression plasmid vectors in Escherichia coli. Methods Enzymol. 185:1437.

15.Sergueev, K., D. Yu, S. Austin, and D. Court. 2001. Cell toxicity caused by products of the $\mathrm{p}(\mathrm{L})$ operon of bacteriophage lambda. Gene 272:227-235.

16.Bateman, A., E. Birney, R. Durbin, S.R. Eddy, K.L. Howe, and E.L. Sonnhammer. 2000. The Pfam protein families database. Nucleic Acids Res. 28:263-266.

Received 1 December 2004; accepted 18 January 2005.

Address correspondence to:

Manabu Nakayama

Kazusa DNA Research Institute

2-6-7 Kazusa-Kamatari, Kisarazu

Chiba 292-0818 Japan

e-mail: nmanabu@kazusa.or.jp 